\title{
Critical phase boundary and finite-size fluctuations in Su-Schrieffer-Heeger model with random inter-cell couplings
}

\author{
Dmitriy S. Shapiro ${ }^{1,2,3},{ }^{*}$ Sergey V. Remizov ${ }^{1,4,5}$, Andrey V. Lebedev ${ }^{1,6}$, Danila V. \\ Babukhin $^{1}$, Ramil S. Akzyanov ${ }^{1,7}$, Andrey A. Zhukov ${ }^{1}$, and Leonid V. Bork ${ }^{1}$ \\ ${ }^{1}$ Dukhov Research Institute of Automatics (VNIIA), 127055 Moscow, Russia \\ ${ }^{2}$ National University of Science and Technology MISiS, 119049 Moscow, Russia \\ ${ }^{3}$ Institute for Quantum Materials and Technologies, \\ Karlsruhe Institute of Technology, 76021 Karlsruhe, Germany \\ ${ }^{4}$ V. A. Kotel'nikov Institute of Radio Engineering and Electronics, \\ Russian Academy of Sciences, Moscow 125009, Russia \\ ${ }^{5}$ Department of Physics, National Research University Higher School of Economics, Moscow 101000, Russia \\ ${ }^{6}$ Moscow Institute of Physics and Technology, 141700, \\ Institutskii Per. 9, Dolgoprudny, Moscow Distr., Russia and \\ ${ }^{7}$ Institute for Theoretical and Applied Electrodynamics, \\ Russian Academy of Sciences, 125412 Moscow, Russia
}

\begin{abstract}
A dimerized fermion chain, described by Su-Schrieffer-Heeger (SSH) model, is a well-known example of 1D system with a non-trivial band topology. An interplay of disorder and topological ordering in the SSH model is of a great interest owing to experimental advancements in synthesized quantum simulators. In this work, we investigate a special sort of a disorder when inter-cell hopping amplitudes are random. Using a definition for $\mathbb{Z}_{2}$-topological invariant $\nu \in\{0 ; 1\}$ in terms of a non-Hermitian part of the total Hamiltonian, we calculate $\langle\nu\rangle$ averaged by random realizations. This allows to find (i) an analytical form of the critical surface that separates phases of distinct topological orders and (ii) finite size fluctuations of $\nu$ for arbitrary disorder strength. Numerical simulations of the edge modes formation and gap suppression at the transition are provided for finite-size system. In the end, we discuss a band-touching condition derived within the averaged Green function method for a thermodynamic limit.
\end{abstract}

\section{INTRODUCTION}

The Su-Schrieffer-Heeger (SSH) model [1, 2] is a onedimensional (1D) tight-binding model of a fermion chain with a certain degree of a dimerization, i.e., with alternating hopping amplitudes. It was applied initially for a description of topological excitations, moving solitons, in trans-polyacetylene molecules that have a doubly degenerate ground state. Later on, the dimerized fermion model was reexamined in condensed matter physics in contexts of topological insulators, fractionalization of quasiparticle charge, and adiabatic spin pump [3-6]. The SSH model describes a connection of geometric Zak phase and band topology in 1D case, where non-trivial edge modes can be formed. The experimental realizations of topological phases in SSH become feasible in such platforms as trapped ultracold atomic gases [7-11] and superconducting qubits [12].

A significant interest is attracted by generalizations of SSH model. They include extensions on two-chain ladders [13], 2D lattice [14], and long-range hopping [15]. This model has a deep connection to driven-dissipative systems described by non-Hermitian Hamiltonians [1618]. As it was systematically studied in Refs. [19-22], disordered versions of SSH chains reveal transitions into topological Anderson insulator phase. An experimental

*Electronic address: shapiro.dima@gmail.com simulation of this phenomenon in ultracold atoms was reported in Ref. [11].

In this work, we provide an analytical calculation of $\mathbb{Z}_{2}$ topological index $\nu$ averaged via central limiting theorem. This solution provides a relation for the critical phase boundary. For finite size system, we provide a formula for fluctuations of the index and study numerically how edge modes evolve when the disorder increases.

The paper is organized as follows. We start from an introducing of the model in Sec. II. In Sec. III we define methods of a calculation $\nu$ in clean system (III A) and in disordered one (IIIB). In Sec. IV we present our results. In the part IV A the analytic formula for $\langle\nu\rangle$ is obtained and in IV B the critical phase boundary and fluctuations are calculated. Results of numerical simulations for finite-size systems are presented in Sec. IV C: phase diagram is analyzed in IV C 1 edge modes wavefunction in IV C 2 , and the gap suppression in IV C 3. In Sec. V we conclude. The averaged Green function is found in Appendix VIA. In Appendix VIB we derive a band-touching condition from the spectral density of states.

\section{MODEL}

The SSH Hamiltonian for a dimerized chain,

$$
H=\sum_{i=1}^{N} u_{i}\left(a_{i}^{\dagger} b_{i}+b_{i}^{\dagger} a_{i}\right)+w \sum_{i=1}^{N-1}\left(a_{i+1}^{\dagger} b_{i}+b_{i}^{\dagger} a_{i+1}\right),
$$


consists of two types of sublattices with fermion orbitals where hopping amplitudes are chosen real. The respective annihilation (creation) operators are $a_{i}\left(a_{i}^{\dagger}\right)$ and $b_{i}\left(b_{i}^{\dagger}\right)$, where $i \in\{1, \ldots, N\}$ and $N$ is total number of dimers. Intra-cell hopping amplitudes (at even bonds) are constant and equal to $w$. Inter-cell amplitudes at odd bonds, $u_{i}$, are random with the average value $\left\langle u_{i}\right\rangle=u$. Random deviations $\delta u_{i}=u_{i}-u$ are uncorrelated at different sites, i.e., $\left\langle\delta u_{i} \delta u_{i^{\prime}}\right\rangle=\delta_{i, i^{\prime}} \gamma^{2}$. Here, $\gamma$ is the disorder strength. This disorder preserves the chiral symmetry of (1), i.e., $S H S=-H$ where $S=\prod_{i=1}^{N}\left(a_{i}^{\dagger} a_{i}-b_{i}^{\dagger} b_{i}\right)$. This symmetry indicates that zero energy modes can exist and a quantum phase transition into a topological Anderson insulator state is possible [23].

The SSH model is known to have two distinct topological phases. They are distinguished by the presence or absence of the midgap edge modes localized at the chain ends. (The energies of these states are exponentially close to $E=0$ in the thermodynamic limit, $N \rightarrow \infty$.) The phases have two distinct topologies of energy bands characterized by $\mathbb{Z}_{2}$-topological invariant $\nu$ that takes two possible values, $\nu=0$ and $\nu=1$. There is a spectral gap, $E_{G}=2|u-w|$, in both of these phases. It means that the topological phase transition, which occurs at the critical point $|w|=|u|$, is accompanied by the band-touching phenomenon.

\section{METHODS}

\section{A. Topological invariant in the clean limit}

In the limit of infinite $N$ and $\gamma=0$, the binary $\nu$ can be formulated in terms of a geometric phase. In order to do that we rewrite the translationary invariant $H$ as an integral over the Brillouin zone with momentum $\mathbf{k} \in[-\pi, \pi]$,

$$
H=\int_{-\pi}^{\pi} \frac{d \mathbf{k}}{2 \pi}\left[\begin{array}{ll}
a_{\mathbf{k}}^{\dagger} & b_{\mathbf{k}}^{\dagger}
\end{array}\right]\left[\begin{array}{cc}
0 & u+w^{-i \mathbf{k}} \\
u+w e^{i \mathbf{k}} & 0
\end{array}\right]\left[\begin{array}{l}
a_{\mathbf{k}} \\
b_{\mathbf{k}}
\end{array}\right] .
$$

The Fourier transform to the momentum space reads as $a_{i}=\int \frac{d \mathbf{k}}{2 \pi} a_{\mathbf{k}} e^{i \mathbf{k} n}$ (and similarly for $b_{i}$ ). The topological index is given as $\nu=\frac{1}{\pi} \varphi_{\mathrm{Zak}}$ where $\varphi_{\mathrm{Zak}}$ is the geometric Zak phase. It is given by an integral over the Brillouin zone of a Berry connection: $\varphi_{\mathrm{Zak}}=(-i) \int_{-\pi}^{\pi}\left\langle\psi_{\mathbf{k}}\left|\partial_{\mathbf{k}}\right| \psi_{\mathbf{k}}\right\rangle d \mathbf{k}$. Eigenfunctions $\left|\psi_{\mathbf{k}}\right\rangle$ correspond to the lower band with the dispersion $\varepsilon_{\mathbf{k}}(u, w)=$ $-\sqrt{u^{2}+w^{2}+2 u w \cos \mathbf{k}}$. They read

$$
\left|\psi_{\mathbf{k}}\right\rangle=\frac{1}{\sqrt{2}}\left[\begin{array}{c}
-e^{-i \operatorname{arctg} \frac{w \sin \mathbf{k}}{u+w \cos \mathbf{k}}} \\
1
\end{array}\right] .
$$

Consequently, the Berry connection is a $2 \pi$-periodic in $k$ function given as $\left\langle\psi_{\mathbf{k}}\left|\partial_{\mathbf{k}}\right| \psi_{\mathbf{k}}\right\rangle=i \frac{w(w+u \cos k)}{2 \varepsilon_{\mathbf{k}}^{2}(u, w)}$. The integral in $\varphi_{\text {Zak }}$ can be performed after the change of variables, $e^{\mathbf{i}}=z$, and integration along the contour $|z|=1$.
There are two poles enclosed by the contour and their residues contribute to $\varphi_{\text {Zak }}$. The first pole is located at $z=0$ and the second one is at $z=-u / w$ if $|u / w|<1$ (or at $z=-w / u$ if $|u / w|>1$ ). After some algebra one finds:

$$
\nu= \begin{cases}0, & |u|>|w| \\ 1, & |u|<|w|\end{cases}
$$

If the first and last elements in the chain have hopping amplitudes $u$, then one has $\nu=0$ for $|u|>|w|$, and $\nu=1$ for $|u|<|w|$.

The value of $\nu=0$ corresponds to trivial phase with an absence of zero modes. Oppositely, $\nu=1$ is related to a topological phase with a presence of non-trivial zero modes. It can be illustrated with the use of secular equation $\operatorname{det} H=0$ for zero energy. It has two complex solutions for momenta $\mathbf{q}_{a, b}= \pm i \ln |w / u|+\pi$ which correspond to $a$ and $b$ sublattices, respectively. We note that in the trivial phase, $|w / u|<1$, zero modes do not exist because wavefunctions would grow exponentially. Oppositely, in the topological phase with $|w / u|>1$ these solutions decay and, consequently, determine wavefunctions of edge states. One of them belongs to $a$-sublattice. It is localized at the left $(n=0)$ edge and has the exponential envelope $\left|\phi_{n}^{(a)}\right\rangle \sim(-1)^{n-1} e^{-(n-1) / \xi}$. Another edge mode is hosted by $b$-sublattice and is located at the opposite edge, $\left|\phi_{n}^{(b)}\right\rangle \sim(-1)^{N-n} e^{-(N-n) / \xi}$. The coherence length is $\xi=\left(\left|\operatorname{Im} \tilde{\mathbf{q}}_{a, b}\right|\right)^{-1}=\frac{1}{\ln |w / u|}$. Of course, in the finite size system these solutions are not exact. As follows from symmetries of the Hamiltonian, equally weighted linear combinations of these exponential solutions can approximate exact wavefunctions of edge modes. The overlap integral of the above solutions determines exponentially small gap between their eigenvalues.

\section{B. Topological invariant in disordered system}

In a system with a disorder, $\gamma \neq 0$, there is no translation invariance and the above method can not be applied. An alternative definition for $\nu$ is based on an auxiliary Aharonov-Bohm phase $\Phi$ introduced for SSH chain closed in a loop [18]. A periodic boundary condition (PBC) is implied in this consideration; it provides a gauge-invariant $\Phi$. By an analogy with the previous case of $\gamma=0$, where we dealt with $2 \pi$-periodic Berry connection, here, the Hamiltonian becomes $2 \pi$-periodic by $\Phi$. This method is based on an analysis of a complex phase of a non-Hermitian part $h$ of the total Hamiltonian represented as $H=h+h^{\dagger}$. Here, the non-Hermitian operator $h$ annihilates $b$-states and creates $a$-states only: $h=\sum_{i=1}^{N} u_{i} a_{i}^{\dagger} b_{i}+w \sum_{i=1}^{N-1} a_{i+1}^{\dagger} b_{i}$. In order to impose PBC one adds the term $w a_{1}^{\dagger} b_{N}$ into $h$ (and $w b_{N}^{\dagger} a_{1}$ into $h^{\dagger}$ ). The new Hamiltonian with $\mathrm{PBC}$ is $h_{\mathrm{PBC}}=h+w a_{1}^{\dagger} b_{N}$. The phase can be gauged into hopping matrix elements 
that is equivalent to a phase drop along the chain. Without loss of generality, we assume that $\Phi$ is dropped at $N$-th bond. This transform changes a complex phase of the matrix element, i.e., one replaces $w \rightarrow w e^{i \Phi}$ in $h$. Finally, the non-Hermitian matrix is introduced,

$$
\mathbf{h}_{i, j}(\Phi)=\delta_{i, j} u_{i}+w\left(\delta_{i, j+1}+e^{i \Phi} \delta_{i, j-N+1}\right) .
$$

It parametrizes the phase dependent Hamiltonian as $h_{\mathrm{PBC}}(\Phi)=\sum_{i, j}^{N} a_{i}^{\dagger} \mathbf{h}_{i, j}(\Phi) b_{j}$. The matrix (5) possesses a desired $2 \pi$-periodicity which provides the alternative definition of the topological invariant:

$$
\nu=\frac{1}{2 \pi i} \int_{0}^{2 \pi} d \Phi \frac{\partial}{\partial \Phi} \ln \left(\operatorname{det} \mathbf{h}_{i, j}(\Phi)\right) .
$$

It can be shown that in the clean limit this definition becomes equivalent to that formulated via the Berry connection.

\section{RESULTS}

\section{A. Averaging via central limiting theorem}

A behavior of the invariant as a function of $w / u$ and $\gamma / u$ depends on a particular disorder realization. In what follows we study how its average by realizations, denoted as $\langle\nu\rangle$, does behave. The average $\langle\mathcal{O}\rangle$ of a quantity $\mathcal{O}$, which depends on the set of $N$ independent random $u_{i}$, is defined as $N$-dimensional integral with infinite limits $\langle\mathcal{O}\rangle=\iint \ldots \int \mathcal{O}\left[u_{1}, . ., u_{N}\right] \prod_{i=1}^{N} \mathcal{P}_{\gamma}\left(u_{i}\right) d u_{i}$. Here, all of $u_{i}$ are weighted with the same probability distribution $\mathcal{P}_{\gamma}(\epsilon)$ normalized to the unity, $\int \mathcal{P}_{\gamma}(\epsilon) d \epsilon=1$. The subscript in $\mathcal{P}_{\gamma}(\epsilon)$ stands for the variance $\gamma$ provided by this distribution, i.e., the relation $\int \epsilon^{2} \mathcal{P}_{\gamma}(\epsilon) d \epsilon-u^{2}=\gamma^{2}$ is implied. For the sake of compactness, we use the function $p_{\gamma}(\epsilon) \equiv \mathcal{P}_{\gamma}(\epsilon-u)$ hereafter that describes random deviations $\delta u_{i}$ around the mean $u$. $\left(p_{\gamma}(\epsilon)\right.$ has the same variance as $\mathcal{P}_{\gamma}(\epsilon)$ but zero first moment).

According to (5) and (6), we find that

$$
\langle\nu\rangle=\langle\theta(1-\xi)\rangle,
$$

where $\theta(x)$ is the Heaviside step function, and the random value $\xi$ is introduced,

$$
\xi=\left(\frac{u}{w}\right)^{N} \prod_{i=1}^{N}\left|1+\frac{\delta u_{i}}{u}\right| .
$$

As shown below, this relation between $\xi$ and products of $u_{i}$ provides a non-trivial result of the averaging.

We start our consideration from the noting that the following identity holds,

$$
\langle\theta(1-\xi)\rangle=\langle\theta(-\ln \xi)\rangle .
$$

Let us think about $\ln \xi$ as of a new random variable. According to (8), $\ln \xi=N \ln \frac{u}{w}+\eta$ where $\eta=\sum_{i=1}^{N} \ln \mid 1+$ $\delta u_{i} / u \mid$. The central limiting theorem can be applied at this step for $\eta$. It says that the sum of independent and identically distributed random variables is normally distributed as

$$
P(\eta)=\frac{1}{\sqrt{2 \pi} z_{2}} \exp \left(-\frac{1}{2 z_{2}^{2}}\left[\eta-N\left(\ln \frac{u}{w}+z_{1}\right)\right]^{2}\right) .
$$

Here, $z_{1}$ and $z_{2}$ are the first and second cumulants of $\eta$. For an arbitrary random distribution $p_{\gamma}(\epsilon)$, their expressions read as

$$
z_{1}=\int \ln (1+\epsilon / u) p_{\gamma}(\epsilon) d \epsilon
$$

and

$$
z_{2}=\int[\ln (1+\epsilon / u)]^{2} p_{\gamma}(\epsilon) d \epsilon-z_{1}^{2} .
$$

Having applied the central limiting theorem, the average (9) is reduced to the integral $\langle\theta(-\ln \xi)\rangle=$ $\int \theta(-\eta) P(\eta) d \eta$. One obtains that $\langle\nu\rangle$ is a continuous function of all Hamiltonian parameters because the step function is smoothed after the integration by $\eta$ with the Gaussian profile $P(\eta)$. The integration is performed straightforward and one arrives at one of central results of this work:

$$
\langle\nu\rangle=\frac{1}{2}\left(1-\operatorname{erf}\left[\sqrt{N} \frac{\ln (u / w)+z_{1}(\gamma / u)}{\sqrt{2 z_{2}(\gamma / u)}}\right]\right) .
$$

This analytical formula for $\langle\nu\rangle$ describes a critical phase boundary and finite-size fluctuations of the invariant near the transition. Thus, having started from the step function with a random argument, we arrived after the averaging at the non-trivial dependence (13) which is valid for a wide range of $\gamma$ and $w$. At finite $N$ the transition between different topological phases, associated with $\langle\nu\rangle=0$ and $\langle\nu\rangle=1$, is smooth due to the averaging. In the thermodynamic limit, $N \rightarrow \infty$, there are no finite size fluctuations and it becomes sharp.

\section{B. Critical phase boundary. Fluctuations of $\mathbb{Z}_{2}$ invariant}

The result (13) allows to obtain the following properties of the phase transition. First, this is the critical phase boundary. It follows from (13) under the condition $\langle\nu\rangle=1 / 2$. Resolving it one finds a critical $w_{0}$ at a given $\gamma$ and $u$. According to (13), it reads:

$$
w_{0}=u e^{z_{1}(\gamma / u)} .
$$

An alternative resolving of this condition with respect to $\gamma$ is complicated because one has to solve a transcendental equation. However, this can be done in the 
limit of weak disorder $\gamma / u \ll 1$ and weak dimerization, $u-w \ll u$, where $u>w>0$. In this limit one finds $z_{1} \approx-\frac{\gamma^{2}}{2 u^{2}}$. Embedding this approximate expression into the Eq. (14) one arrives at the critical disorder strength,

$$
\gamma_{0}=\sqrt{2 u(u-w)} .
$$

Similarly to the clean limit, the topological transition driven by the disorder, which occurs at $\gamma=\gamma_{0}$, is accompanied by a gap closing as well. The gap closing can be shown analytically in the limit of $u-w \ll u$ via a calculation of the density of states within first Born approximation (see Appendix VI).

Second, for the binary quantity $\nu$ we immediately find that finite size fluctuations of $\Delta \nu=\nu-\langle\nu\rangle$ are given by

$$
\left\langle\Delta \nu^{2}\right\rangle=\langle\nu\rangle(1-\langle\nu\rangle) .
$$

In the limits of weak disorder and dimerization mentioned above, one finds $z_{2} \approx \frac{\gamma^{2}}{u^{2}}$. In this case, the fluctuations read

$$
\left\langle\Delta \nu^{2}\right\rangle=\frac{1}{4}\left(1-\operatorname{erf}^{2}\left[\sqrt{\frac{N}{2}}\left(\frac{u-w}{\gamma}-\frac{\gamma}{2 u}\right)\right]\right) .
$$

The width $\Delta \gamma$ of the fluctuational region near the critical value $\gamma_{0}$, when other parameters are constant, is estimated as

$$
\Delta \gamma \sim \frac{u}{\sqrt{N}}
$$

We note that only the size of the system appears in this estimation while the dimerization parameter does not. This means that finite-size fluctuations near the critical surface are usually not small.

\section{Numerical simulations}

\section{Phase diagram}

The formula (13) demonstrates a good agreement with the data found after the numerical averaging. Hereafter, we assume flat distribution in $u_{i} \in[u-\sqrt{3} \gamma ; u+\sqrt{3} \gamma]$ with

$$
p_{\gamma}(\epsilon)=\frac{1}{2 \sqrt{3} \gamma} \theta(\sqrt{3} \gamma-|\epsilon|) .
$$

As demonstrated in Fig. 1, the theoretical dependence (13) (blue curve) matches with data of the simulation (red dots) for the chain with $N=100$ dimers. The agreement is observed in a domain of strong disorder, $\gamma \gtrsim u$, as well.

If $w$ and $\gamma$ are varied at a constant $u$, then one arrives at a phase diagram of the disorder driven transition. It is shown in the Fig. 2 where the gap value is plotted for the system of $N=300$ dimers where PBC are imposed.

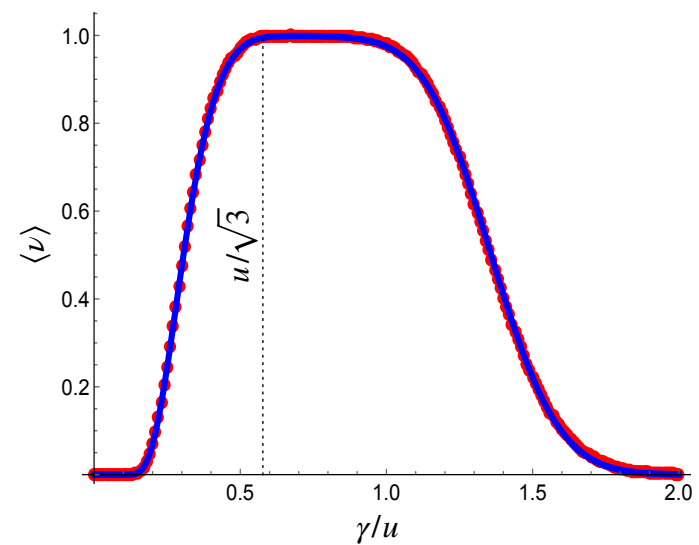

FIG. 1: Topological invariant averaged by disorder realizations $\langle\nu\rangle$ (red dots) as a function of the disorder strength, $\gamma$. The simulation is performed for the chain of $N=100$ dimers, $w / u=0.95$, and $15 \times 10^{3}$ random distributions for $u_{i}$. Blue line: theoretical dependence given by Eq. (13). The vertical dashed line is a character value of $\gamma$ that separates domains of weak and strong disorder.

The data shown correspond to a particular disorder realization. Bright red areas correspond to a finite gap value and blue ones to a suppressed gap. Joined black dots represent the boundary between trivial $(\nu=0)$ and topological $(\nu=1)$ phases for the particular realization of $H$. The red curve determines the critical phase boundary $w_{0}$ as a function of $\gamma$, see Eq. (14), where the exponent has the following explicit form:

$$
z_{1}=-1+\frac{u}{2 \sqrt{3} \gamma} \ln \frac{u+\sqrt{3} \gamma}{|u-\sqrt{3} \gamma|}+\frac{1}{2} \ln \left|1-\frac{3 \gamma^{2}}{u^{2}}\right| .
$$

The function is found after the integration in (11) with the flat distribution.

\section{Edge modes}

Here, we analyze an evolution of the edge modes wavefunction of the chain Hamiltonian (1) when $\gamma$ increases. In numerical simulations, we consider the eigenstates $\psi_{\sigma}\left(q E_{\min }\right)$ from upper and lower bands that have energies closest to $E=0$. Here, $\sigma=a, b$ stands for the sublattice index and the minimal energies are $q E_{m}$ where $E_{\min }=\min \left|E_{j}\right|$ and $q= \pm 1$ due to particle-hole symmetry of $H$. For a particular realization of the disorder we calculate the wavefunction

$$
|\psi|^{2}=\sum_{\sigma=a, b ; q= \pm 1}\left|\psi_{\sigma}\left(q E_{\min }\right)\right|^{2}
$$

where a trace over $q$ - and $\sigma$-index is taken. On a next step, the averaging of (21) is performed.

In Fig. 3 we present the data for $\left\langle|\psi|^{2}\right\rangle$ with the averaging over 100 disorder realizations with $N=100$ dimers and $w / u=0.95$. One can see that edge modes appear at 


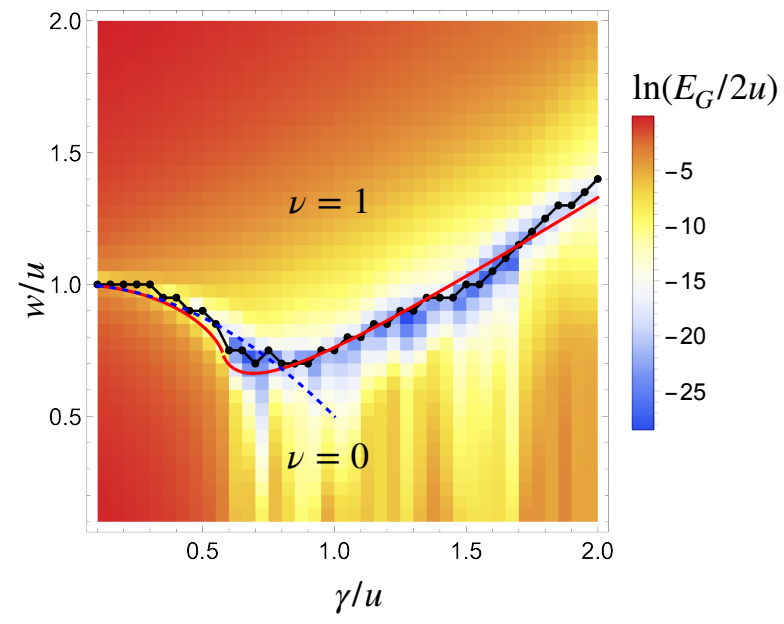

FIG. 2: Phase diagram of the topological transition for a particular disorder realization. Density plot: the gap value in the logarithmic scale, $\ln \left(E_{G} / 2 u\right)$, as a function of $\gamma$ and $w$ in the system of $N=300$ dimers with PBC. Black joined dots: the boundary between trivial and topological phases. Red curve: critical phase boundary given by Eqs. (14) and (20). Blue dashed line: critical $w=u \sqrt{1-\frac{\gamma^{2}}{2 u^{2}}}$ found for weak dimerization limit (see Eq. 15) where the Born approximation is justified.

$\gamma$ above $\gamma_{0}$ where $\langle\nu\rangle$ (red dots) saturates to the unity. Further increase of $\gamma / u \gtrsim 1$ reveals a disruption of localized modes and a reentrance into the non-topological phase with the saturation of the averaged invariant to $\langle\nu\rangle=0$. The smooth decrease of $\langle\nu\rangle$ from the unity to zero means that the topological invariant is strongly fluctuating and is sensitive to a particular random realization.

\section{Energy gap suppression}

Similarly to the clean limit, the disorder driven topological transition is accompanied by the gap closing. This effect is described analytically in the limit of $u-w \ll u$ via a calculation of the density of states within first Born approximation (see Appendix VI). In simulations for a finite-size system (see Fig. 4) we observe a strong suppression of the gap, $E_{G}=2 E_{m}$, which is decreased by five orders in the magnitude (blue dots). The data are shown for the chain of $N=300$ dimers and averaging over 100 realizations. This suppression corresponds to the bandtouching phenomenon in the thermodynamic limit. At the same time, the averaged $\langle\nu\rangle$ changes smoothly from 0 to 1 (red dots) near the critical point $\gamma=\gamma_{0}$. The relative width of this transition, which is of the order of $10 \%$, is in agreement with the estimation (18) that predicts a weak power-law decay of width with $N$.

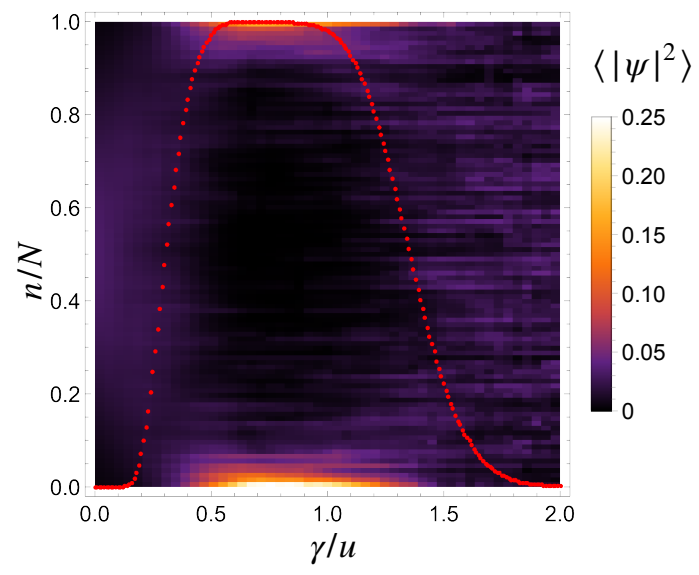

FIG. 3: The wavefunction given by Eq. (21) averaged by 100 realizations. Simulation is performed for the chain Hamiltonian (1) with $N=100$ dimers and $w / u=0.95$. Density plot for $\left\langle|\psi|^{2}\right\rangle$ shows a formation of edge states (red spots near $n=0$ and $n=N)$ when the averaged topological invariant $\langle\nu\rangle$ (red dots) saturates to unity. Decrease of $\langle\nu\rangle$ when $\gamma / u \gtrsim 1$ corresponds to a disruption of the edge modes and an onset of a randomly distributed wavefunction.

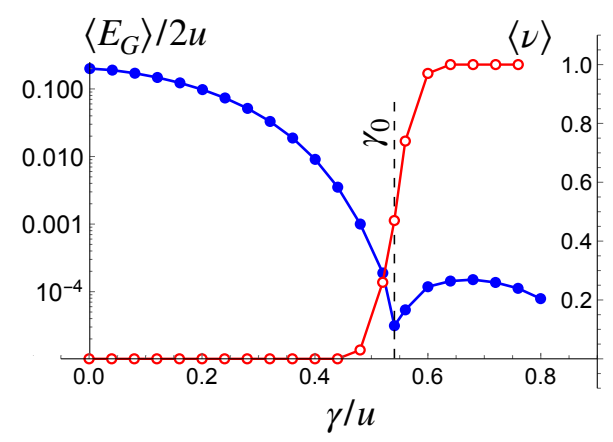

FIG. 4: Topological phase transition driven by the disorder and suppression of the gap. Numerical data for $\left\langle E_{G}\right\rangle$ (blue dots, left axis) and $\langle\nu\rangle$ (red dots, right axis) averaged over 100 disorder realizations as a function of the disorder strength, $\gamma$. The simulated system involves $N=300$ dimers with PBC and $w / u=0.8$. The critical value of $\gamma_{0}$ corresponds to the transition between trivial $(\nu=0)$ and topological $(\nu=1)$ phases and to the minumum of the gap value.

\section{DISCUSSION AND OUTLOOK}

To conclude, we studied theoretically topological transitions in finite-size disordered SSH model. Our findings were motivated by state-of-the-art experiments where topologically ordered phases were observed in artificial dimerized chains. In this work, we derived an analytic formula for $\mathbb{Z}_{2}$-topological invariant $\nu$ and its fluctuations averaged by an ensemble. The approach is based on the central limiting theorem and a non-Hermitian Hamiltonian. In particular, this method gives an exact result for the critical surface at an arbitrary strength of the disorder. Our work is complementary to previous stud- 
ies of topological phases in disordered chains [19-22]. A particular case of random inter-cell tunnelling rates was considered here, however, the results can be extended for a more general forms of the disorder that preserve chiral symmetry. We also provided a detailed comparison of our findings with numerical simulations.

\section{Acknowledgments}

The reported study was supported by Russian Foundation for Basic Research (RFBR) according to the research project № 20-37-70028. D.S.S. also acknowledges the support by RFBR research project № 20-52-12034, and by DFG Grant No. MI 658/13-1 within a joint DFGRSF project.

\section{APPENDIX}

\section{A. Averaged Green function within Born approximation}

Consider a retarded propagator, which has $2 N \times 2 N$ matrix structure in the coordinate $\left(n, n^{\prime}\right)$ and sublattice $(\sigma)$ spaces,

$$
\begin{aligned}
& {\left[G_{n, n^{\prime}}\left(t-t^{\prime}\right)\right]_{\sigma, \sigma^{\prime}}=} \\
& \quad\left[i \delta_{n, n^{\prime}} \delta_{\sigma, \sigma^{\prime}} \delta\left(t-t^{\prime}\right) \partial_{t^{\prime}}-\delta\left(t-t^{\prime}\right)\left[\mathcal{H}_{n, n^{\prime}}\right]_{\sigma, \sigma^{\prime}}\right]^{-1}
\end{aligned}
$$

for the system with the Hamiltonian (1). The matrix $\left[\mathcal{H}_{n, n^{\prime}}\right]_{\sigma, \sigma^{\prime}}=\left\langle n, \sigma|H| n^{\prime}, \sigma^{\prime}\right\rangle$ is a projection of (1) on the single-particle basis $|n, \sigma\rangle$ where states are defined on $n$ th site and sublattice index $\sigma=a, b$. As long as the system is stationary, we use a Fourier transform by the time, i.e. $\partial_{t} \rightarrow-i \omega$. Let us represent the Hamiltonian matrix $\mathcal{H}=\mathcal{H}^{(0)}+\mathcal{V}$, i.e. as a sum of the translational invariant part

$$
\mathcal{H}_{n, n^{\prime}}^{(0)}=u \delta_{n, n^{\prime}} \sigma_{x}+w\left(\delta_{n, n^{\prime}+1} \sigma_{+}+\delta_{n+1, n^{\prime}} \sigma_{-}\right),
$$

and the part with the disorder, $\mathcal{V}_{n, n^{\prime}}=\epsilon_{n} \delta_{n, n^{\prime}} \sigma_{x}$ that is considered as a perturbation. The sublattice indices are encoded by Pauli matrices $\sigma_{x}, \sigma_{+}=\frac{1}{2}\left(\sigma_{x}+i \sigma_{y}\right)$ and $\sigma_{-}=\frac{1}{2}\left(\sigma_{x}-i \sigma_{y}\right)$. The Fourier transformed propagator (A1) is expanded in series by $\mathcal{V}$ :

$$
G_{n, n^{\prime}}(\omega)=\mathcal{G}_{n, n^{\prime}}(\omega)+\mathcal{G}_{n, k}(\omega) \sum_{q=1}^{\infty}\left[(\mathcal{V G}(\omega))^{q}\right]_{k, n^{\prime}}
$$

Here, the matrix $\mathcal{G}_{n, n^{\prime}}(\omega)$ is the retarded propagator for the clean system: $\mathcal{G}_{n, n^{\prime}}(\omega)=\left[\delta_{n, n^{\prime}}(\omega+i \alpha) \sigma_{0}-\right.$ $\left.\mathcal{H}_{n, n^{\prime}}^{(0)}\right]^{-1}$ where $\alpha$ is a positive infinitesimal frequency. Performing a discrete Fourier transform and using the representation of the Hamiltonian from (2), on finds $\sum_{n, n^{\prime}} e^{-i k n-i k^{\prime} n^{\prime}} \mathcal{G}_{n, n^{\prime}}(\omega)=2 \pi \delta\left(\mathbf{k}-\mathbf{k}^{\prime}\right) \mathcal{G}_{\mathbf{k}}(\omega)$. Here, the bare Green function is defined for a momentum $\mathbf{k}$ in the Brillouin zone. It has a two-dimensional structure in $\sigma$ space:

$$
\begin{aligned}
& \mathcal{G}_{\mathbf{k}}(\omega ; u, w)= \\
& \quad=\frac{(\omega+i \alpha) \sigma_{0}+(u+w \cos \mathbf{k}) \sigma_{x}+w \sin \mathbf{k} \sigma_{y}}{(\omega+i \alpha)^{2}-\epsilon_{\mathbf{k}}^{2}(u, w)} .
\end{aligned}
$$

Let us return back to the Green function in the coordinate space (A3). The averaging of $G_{n, n^{\prime}}$ by disorder realizations makes it translational invariant. Below we perform this calculation in a first order self-energy (Born approximation) where one takes into account only Focktype diagram [24]. The averaged Green function does not include crossed line and "rainbow" diagrams; it is represented as follows:

$$
\left\langle G_{n, n^{\prime}}\right\rangle=\mathcal{G}_{n, n^{\prime}}+\mathcal{G}_{n, k} \sum_{q=1}^{\infty}\left[\Sigma_{k, m} \mathcal{G}_{m, n^{\prime}}\right]^{q} .
$$

The straightforward resummation in (A5) yields the Green function in the first Born approximation:

$$
\left\langle G_{\mathbf{k}}(\omega)\right\rangle=\left[\mathcal{G}_{\mathbf{k}}^{-1}(\omega)-\Sigma_{\mathbf{k}}^{(\mathrm{BA})}(\omega)\right]^{-1} .
$$

Here, the self-energy is given by $\Sigma_{n, n^{\prime}}^{(\mathrm{BA})}=$ $\left\langle\mathcal{V}_{n, k} \mathcal{G}_{k, m} \mathcal{V}_{m, n^{\prime}}\right\rangle$. After the averaging one finds that the self-energy is local in the real space and reads:

$$
\Sigma_{n, n^{\prime}}^{(\mathrm{BA})}(\omega ; u, w)=\gamma^{2} \delta_{n, n^{\prime}} \sigma_{x} \mathcal{G}_{n, n}(\omega ; u, w) \sigma_{x} .
$$

Here, $\mathcal{G}_{n, n}=\int_{-\pi}^{\pi} \mathcal{G}_{\mathbf{k}} \frac{d \mathbf{k}}{2 \pi}$. Odd terms in $\mathbf{k}$ cancel out under the integration and we have the following structure of the self-energy in $\sigma$-space:

$$
\Sigma_{\mathbf{k}}^{(\mathrm{BA})}(\omega ; u, w)=\gamma^{2}\left[f(\omega ; u, w) \sigma_{0}+g(\omega ; u, w) \sigma_{x}\right] .
$$

Here,

$$
f(\omega ; u, w)=\int_{-\pi}^{\pi} \frac{\omega+i \alpha}{(\omega+i \alpha)^{2}-\epsilon_{\mathbf{k}}^{2}(u, w)} \frac{d \mathbf{k}}{2 \pi} .
$$

and

$$
g(\omega ; u, w)=\int_{-\pi}^{\pi} \frac{u+w \cos \mathbf{k}}{(\omega+i \alpha)^{2}-\epsilon_{\mathbf{k}}^{2}(u, w)} \frac{d \mathbf{k}}{2 \pi} .
$$

We observe that functions $f$ and $g$ renormalize the frequency and hopping element $u$ as follows:

$$
\omega \rightarrow \omega-\gamma^{2} f(\omega ; u, w)
$$

and

$$
u \rightarrow u+\gamma^{2} g(\omega ; u, w)
$$


Finally, we find that the averaged Green function is represented via the bare Green function (A4) as follows:

$$
\begin{aligned}
& \left\langle G_{\mathbf{k}}(\omega ; u, w, \gamma)\right\rangle= \\
& =\mathcal{G}_{\mathbf{k}}\left(\omega-\gamma^{2} f(\omega ; u, w) ; u+\gamma^{2} g(\omega ; u, w), w\right) .
\end{aligned}
$$

The renormalization in (A13) allows to construct a selfconsistent Born approximation procedure. We leave this issue beyond the scope of our consideration.

\section{B. Band-touching condition}

We address the limit of weak dimerization, $|u-w| \ll u$, when the gap in the clean limit is small compared to the bandwidth. The low-energy modes reside close to the momentum $\mathbf{k}= \pm \pi$. We approximate the spectrum near this point reads as $\epsilon_{\mathbf{q}}(\Delta, u)=\sqrt{\Delta^{2}+u^{2} \mathbf{q}^{2}}$; we introduced here the momentum counted from the edge of the Brillouin zone, $\mathbf{q}=\mathbf{k}-\pi$, and the dimerization parameter $\Delta=u-w$ which is small compared to $u$. We note that an exact calculation of $g$ via the contour integrals with $z=e^{i \mathbf{k}}$ gives $g=0$ at $\omega=0$ and $\Delta<0$. Consequently, the band-touching condition is only possible for $\Delta>0$ which is assumed hereafter.

Calculation of the integrals (A9) and (A10) is reduced to an integration of a very narrow Lorentian peak near $\mathbf{q}=0$ within the approximation indicated. We find that for $\omega=0$, that corresponds to local Green function at the midgap, the integrals are

$$
\begin{aligned}
f(\Delta, u) \approx-\int_{-\infty}^{\infty} \frac{i \alpha}{\mathbf{q}^{2}+\frac{\Delta^{2}+\alpha^{2}}{u^{2}}} \frac{d \mathbf{q}}{2 \pi u^{2}} & = \\
& =\frac{-i \alpha}{2 u \sqrt{\Delta^{2}+\alpha^{2}}}
\end{aligned}
$$

and

$$
\begin{aligned}
g(\Delta, u) \approx-\int_{-\infty}^{\infty} \frac{\Delta}{\mathbf{q}^{2}+\frac{\Delta^{2}+\alpha^{2}}{u^{2}}} & \frac{d \mathbf{q}}{2 \pi u^{2}}= \\
& =\frac{-\Delta}{2 u \sqrt{\Delta^{2}+\alpha^{2}}} .
\end{aligned}
$$

The diagonal component of the Green function at coincident coordinates near $\omega=0$ reads:

$$
\mathcal{G}^{\text {(diag) }}=f\left(\omega-\gamma^{2} f(\Delta, u) ; u+\gamma^{2} g(\Delta, u), w\right)
$$

It has the following form

$$
\begin{aligned}
& \mathcal{G}^{(\operatorname{diag})}(\omega) \approx \\
& \approx-\int_{-\infty}^{\infty} \frac{\omega-\gamma^{2} f(\Delta, u)+i \alpha}{\mathbf{q}^{2}+\frac{\left(\Delta+\gamma^{2} g(\Delta, u)\right)^{2}-\left(\omega-\gamma^{2} f(\Delta, u)+i \alpha\right)^{2}}{u^{2}}} \frac{d \mathbf{q}}{2 \pi u^{2}}= \\
& =-\frac{\omega-\gamma^{2} f(\Delta, u)+i \alpha}{2 u \sqrt{\left(\Delta+\gamma^{2} g(\Delta, u)\right)^{2}+\left(\omega-\gamma^{2} f(\Delta, u)+i \alpha\right)^{2}}}
\end{aligned}
$$

The imaginary part of the Green function allows to obtain the spectral density of states as

$$
\rho(\omega)=\frac{-1}{\pi} \operatorname{Im} \mathcal{G}^{(\mathrm{diag})}(\omega)
$$

For the midgap energy, i.e., $\omega=0$, one finds:

$$
\rho(0)=\frac{\alpha\left(1+\frac{\gamma^{2}}{2 u \Delta}\right)}{2 \pi u \sqrt{\left(\Delta-\frac{\gamma^{2} \theta(\Delta)}{2 u}\right)^{2}+\alpha^{2}\left(1+\frac{\gamma^{2}}{2 u \Delta}\right)^{2}}} .
$$

Here, we use approximate form of $f$ and $g$ from (A14) and (A15). The midgap density of states given by (A19) has a singularity when the band-touching condition holds:

$$
\Delta-\frac{\gamma^{2}}{2 u}=0
$$

The equation is resolved as $\gamma_{0}^{(\mathrm{BA})}=\sqrt{2 u(u-w)}$ where $u>w>0$. In other words, the critical disorder strength found after first Born approximation reproduces the result (15) derived via central limiting theorem.
[1] W. P. Su, J. R. Schrieffer, and A. J. Heeger, Phys. Rev. Lett. 42, 1698 (1979).

[2] W. P. Su, J. R. Schrieffer, and A. J. Heeger, Phys. Rev. B 22, 2099 (1980).

[3] X.-L. Qi and S.-C. Zhang, Rev. Mod. Phys. 83, 1057 (2011).

[4] X.-L. Qi, T. L. Hughes, and S.-C. Zhang, Nature Physics 4, 273 (2008).

[5] D.-H. Lee, G.-M. Zhang, and T. Xiang, Phys. Rev. Lett. 99, 196805 (2007).
[6] L. Fu and C. L. Kane, Phys. Rev. B 74, 195312 (2006).

[7] M. Atala, M. Aidelsburger, J. T. Barreiro, D. Abanin, T. Kitagawa, E. Demler, and I. Bloch, Nature Physics 9, 795 (2013).

[8] M. Leder, C. Grossert, L. Sitta, M. Genske, A. Rosch, and M. Weitz, Nature Communications 7, 13112 (2016).

[9] M. Lohse, C. Schweizer, O. Zilberberg, M. Aidelsburger, and I. Bloch, Nature Physics 12, 350 (2016).

[10] D. Xie, W. Gou, T. Xiao, B. Gadway, and B. Yan, npj Quantum Information 5, 1 (2019). 
[11] E. J. Meier, F. A. An, A. Dauphin, M. Maffei, P. Massignan, T. L. Hughes, and B. Gadway, Science 362, 929 (2018).

[12] I. S. Besedin, M. A. Gorlach, N. N. Abramov, I. Tsitsilin, I. N. Moskalenko, A. A. Dobronosova, D. O. Moskalev, A. R. Matanin, N. S. Smirnov, I. A. Rodionov, A. N. Poddubny, and A. V. Ustinov, Phys. Rev. B 103, 224520 (2021).

[13] A. A. Nersesyan, Phys. Rev. B 102, 045108 (2020).

[14] D. Obana, F. Liu, and K. Wakabayashi, Phys. Rev. B 100, 075437 (2019).

[15] H.-C. Hsu and T.-W. Chen, Phys. Rev. B 102, 205425 (2020).

[16] M. S. Rudner and L. S. Levitov, Phys. Rev. Lett. 102, 065703 (2009).

[17] S. Lieu, Phys. Rev. B 97, 045106 (2018).

[18] Z. Gong, Y. Ashida, K. Kawabata, K. Takasan, S. Higashikawa, and M. Ueda, Phys. Rev. X 8, 031079 (2018).

[19] A. Altland, D. Bagrets, L. Fritz, A. Kamenev, and H. Schmiedt, Phys. Rev. Lett. 112, 206602 (2014).

[20] A. Altland, D. Bagrets, and A. Kamenev, Phys. Rev. B 91, 085429 (2015).

[21] I. Mondragon-Shem, T. L. Hughes, J. Song, and E. Prodan, Phys. Rev. Lett. 113, 046802 (2014).

[22] L. Li, Z. Xu, and S. Chen, Phys. Rev. B 89, 085111 (2014).

[23] S. Ryu, A. P. Schnyder, A. Furusaki, and A. W. W. Ludwig, New Journal of Physics 12, 065010 (2010).

[24] A. Altland and B. D. Simons, Condensed matter field theory (Cambridge university press, 2010). 